\title{
How Does Interactive Social Support in Healthcare Communities Promote Customer Well-Being?
}

\author{
Yao Tang \\ School of Economics and Management \\ Beihang University \\ Beijing, People's Republic of China \\ flyingflag@126.com \\ Zhang Qi \\ School of Economics and Management \\ Beihang University \\ Beijing, People's Republic of China \\ 9fengzhi@163.com
}

\author{
Qiu Qi \\ College of Business Administration \\ Capital University of Economics and Business \\ Beijing, People's Republic of China \\ qqbosanova@hotmail.com \\ Zheng Qiuying * \\ School of Management \\ Beijing University of Chinese Medicine \\ Beijing, People's Republic of China \\ 13466379159@163.com
}

\begin{abstract}
This study developed the kinds of social support patients actually obtain on Internet. With data collected from published posts in online health care communities, the authors identify three kinds of support (emotional support, informational support, and companionship) that underlie online social support in health care. Theoretically, the authors constructed a conceptual model of the relationship between online social support and individual's quality of life. The model illustrated that informational, emotional and companionship support affect patients' quality of life in descending order of importance. In particular, online social support as a whole should be encouraged and received by users in online health care communities as they tend to be associated with higher patient's quality of life.
\end{abstract}

Keywords-online; quality of life; social support; healthcare

\section{INTRODUCTION}

China has made great achievements in many aspects including health care system reform. There have been problems, however, in the past economic development, the major problems are inadequate state investment, imbalance in the distribution of medical resources, and variant quality of medical service. Those have become hot issues in China's medical service sector and even the whole nation. The existing health care service cannot fundamentally meet people's needs, and even disputes often occur due to the poor quality of health care service. In short, there are prominent contradictions between contemporary Chinese health care level and economic and social development, and between Chinese health care level and satisfying the needs of people's health management.

In China today, cardiopathy, cancer, cerebra vessels disease and other chronic diseases present the trend of multiple and high incidence. These diseases present the characteristics of long treatment cycle, slow effect, and longterm medication therapy, resulting in patients' negative emotions of varying degrees, and even losing confidence and courage in future life. In addition, a number of patients with AIDS, hepatitis B, tuberculosis, leprosy and other infectious diseases cannot be adequately treated subject to unsatisfactory health care conditions and technical means. Moreover, patients with infectious diseases in reality are rejected and excluded by the society for different reasons, in different ways, to varying degrees because of prejudice and misunderstanding.

Patients who suffered from social exclusion may have negative feelings and behavior; however, it is one of the basic human needs to maintain a stable and good relationship with others, since Chinese culture emphasizes interdependence more than western culture. Psychological needs of the patients, however, cannot be satisfied due to social exclusion of the public in reality, Internet has become one of significant platforms for them to get social support. Internet will not substitute other communication tools but in combination of them and extends the scope of social networks [1]. Rheingold explored the network communication, and found that social coalition online might establish feelings among people, which formed the interpersonal relationship network in the virtual space [2].

In recent years, with the rapid development of Internet in China, many patients have established their own online communities, which are mainly used to ask for social support. Particularly, Internet has been changing the way people learn about health and illness [3]. Internet presents characteristics as convenience, accessibility, anonymity, without interference, and interaction, so virtual community has great potential as a safer platform for disease sufferers to engage in nonthreatening and supportive communication [4]. Patients who meet online could develop friendly and comforting relations and provide each other with various forms of social support. The resulting trust generally increases an individual's willingness to disclose details of his or her disease and experiences [5].

This article is organized as follows: First, we review theory relevant to the social support. Next, we discuss the 
findings of our empirical study of online social support by employing the method of content analysis and initially understand quality of life as the outcome of online social support. Finally, we then develop a model to in-depth describe interrelations between online social support and quality of life.

\section{THEORETICAL REVIEW AND HYPOTHESIS}

\section{A. Social Support}

The influence of social relations on well-being has long been a subject of study across disciplines [6]. Specifically in health care sector, social support can reduce patients' depression, helplessness, anxiety and negative thoughts about themselves and further influence patient well-being and life satisfaction, since patient health conditions are closely related to emotions [7-14]. A direct positive relationship between social support and people's health has been recognized in the research history. There are published reviews on social support in coronary heart disease, stroke, cancer or diabetes, spinal cord injury [15-19]. From conceptual aspect, explained that the belief of supportive interactions among people is protective against the health consequences of life stress in his well-known Presidential Address and resulting article [20]. Following Cobb's (1976) findings, researchers across disciplines have been studying the social support of individuals from different perspectives.

\section{B. Quality of Life}

In recent years, health-related quality of life is viewed as a vital indicator of health care service performance not only by service marketers but also by medical experts. First, service marketers find that quality of life as a social marketing concept is important to business success [21]. In recent years, service performance is increasingly being measured in terms of social as well as financial outcomes [22-24]. Quality of life does not compete with the more traditional financial and growth-oriented objectives of marketing; rather, it enhances our understanding of the potential of marketing activities. In this case, such understanding is through increased knowledge of the impact that service provision has on customer well-being and, by extension, on societal well-being. Marketers can, therefore, positively influence the lives of consumers through the quality-of-life concept [25-26]. Second, quality of life is an important aspect of medical experts' approach to treatment. The European Organization for Research and Treatment of Cancer (EORTC) stated its aim as "to conduct, develop, coordinate, and stimulate laboratory and clinical research in Europe to improve the management of cancer and related problems by increasing not only the survival rate, but also patients' quality of life." Therefore, medical experts developed different versions of quality life scales to measure health care service performance.

In service literatures, McGill version of quality of life attracted scholar's attention due to its parsimony and convenience. Comparing with SF-36's eight dimensions, McGill version of quality of life only comprises four domains: physical, psychological, existential, and support
[27]. The physical domain concerns the individual's most problematic physical symptoms, such as fatigue, pain, and weakness. The psychological domain concerns feelings regarding being depressed, nervous or worried, sadness, and fear of the future. The existential domain concerns an individual's belief about their life, including the belief that life is meaningful and worthwhile, and that goals are achievable, how they feel about themselves, and whether they have a sense of control over life. Support is concerned about feeling supported and cared for. This study selects McGill version of quality of life as the final indicator to measure online social support service performance. However, support dimension is erased from the original scale in present study since it is conceptually close to social support, the antecedent which we are interested in. According to the analysis above, thus we propose the following hypothesis:

H1a: Emotional social support will positively influence patient's physical quality of life.

H1b: Emotional social support will positively influence patient's psychological quality of life.

H1c: Emotional social support will positively influence patient's existential quality of life.

$\mathrm{H} 2 \mathrm{a}$ : Informational social support will positively influence patient's physical quality of life.

$\mathrm{H} 2 \mathrm{~b}$ : Informational social support will positively influence patient's psychological quality of life.

$\mathrm{H} 2 \mathrm{c}$ : Informational social support will positively influence patient's existential quality of life.

H3a: Companionship social support will positively influence patient's physical quality of life.

H3b: Companionship social support will positively influence patient's psychological quality of life.

H3c: Companionship social support will positively influence patient's existential quality of life.

\section{MethodologiES}

To address the research questions, we investigated a large number of online health care communities on the Internet. Hepatitis B is selected as the research setting due to the following reasons: (1) Hepatitis B is a common pandemic infectious disease in China. According to the statistics of Word Health Organization, there are about 350 million hepatitis B virus (HBV) carriers all over the world, and that Mainland China is a high incidence area of HBV infection. The survey in the scope of China also demonstrated that about $60 \%$ of the total population had infected with HBV, of which $80 \%$ are generally asymptomatic with normal liver function. Therefore, all the people with HBV but no symptoms could only be called the carriers, who perhaps account for nearly 93 million in Mainland China, namely one carrier among every 14 Chinese people [28]; (2) HBV patients also face social discrimination and they had to tackle a broad range of social exclusions in employment, day-today living, romantic relations and other aspects of life and work. HBV patients have become typical representatives of vulnerable members because of the disease; (3) AntiHepatitis B Forum is one of the most popular and most active online forums as it has the largest number of members and published posts among all Chinese online health care 
communities (see Table 2). Consequently, in order to investigate online social support among Chinese patients, it's appropriate to take Anti-Hepatitis B Forum as the research setting.

First of all, we captured posts published by online health care community members in the relevant sectors of AntiHepatitis B Forum as samples. Based on the statistics concluded by Anti-Hepatitis B Forum itself, there were over 65,000 themes including numerous posts in total shown in more than 1,300 website pages with an average of around 50 themes in each page. Hereby, we adopt the method of equidistant sample to collect data, namely from the first page to the end, the total of 25 pages including 1,400 themes as samples were extracted at 50-page intervals. Finally, 1,368 themes were finally obtained with 32 invalid themes eliminated.

Then first, four authors read the posts independently at least 2 times and coded all data into a number of categories according to the traditional social support theoretical literatures [29-30]. These categories are (1) emotional support, (2) informational support and (3) companionship (Tichon and Shapiro 2003). When there was disagreement among coders, discussion took place until agreement was reached. It needs specially to note that although instrumental support as provision of material or financial aid, or services was identified one component of social support discussed in the traditional literatures [31-32], it is debatable whether it should be measured in research that focuses on online communities (Bambina 2007) and there are no examples available of this type from our online samples, as it requires individual to reveal his or her real-life identity. Therefore, four authors unanimously agree to eliminate this domain from the categories of online social support in our research.

Our second task was to quantitatively analyze the relationship between online social support categorical variables and patients' quality of life. The study design employed an online survey approach that collected data from Anti-Hepatitis B Forum. We first contacted the forum administrators to get their help to publish a questionnaire link on the top of the website pages to invite members to participate our survey. To encourage more people to fill out the questionnaire, an online lottery was offered as gift to every participant. Finally, a sample of $265 \mathrm{HBV}$ patients or members from Anti-Hepatitis B Forum was randomly selected over three months to participate in the study. In the questionnaire, two separate scales were used to measure each of the four dimensions of online social support and each of the three dimensions of quality of life respectively. The online social support scale with 18 items was constructed through combining two scales developed by Schwarzer and Schulz and Liang and Wei [33]. The items were modified to fit the specific research context and the purpose of measuring Chinese people's online social support actions in health care communities. Moreover, the quality of life items were adapted from McGill Quality of Life Questionnaire, which consists of 14 items constructing physical, psychological, and existential subscales, and originally has been developed to assess QOL of terminal cancer patients.

\section{ANALYSES AND RESULTS}

Analysis was based on a partial disaggregation approach, in which scale items were combined into composites to reduce random error, while retaining the multiple indicator approach of structural equation modeling [34]. Where possible, at least three composite indicators were created per latent construct, as is the recommended approach in the literature (e.g., Hau and Marsh 2004). Fit for the structural model was good $(\chi \square / \mathrm{df}=2.183, \quad \mathrm{p}<.00, \quad \mathrm{RMR}=0.038$, $\mathrm{CFI}=0.947, \mathrm{RMSEA}=0.048)$ (see Table 1$)$.

As shown in Table 2, the results indicate that emotional support had a moderate to large influence on physical, psychological, and existential quality of life $(\beta=0.212$, $\square .=0.335, \beta=0.274$, respectively). Informational support was the dominant direct antecedents of physical, psychological, and existential quality of life $(\beta=0.569, \square .=0.258, \beta=0.175$, respectively). Additionally, companionship also had a large influence on physical, psychological, and existential quality of life $(\beta=0.268, \square=0.357, \beta=0.329$, respectively). Thus, generally as we supposed, online social support impacted three dimensions of quality of life in moderate to large degree, and all 9 hypothesis have been supported statistically.

TABLE I. MEASuREMENT Model Results

\begin{tabular}{|c|c|c|c|c|c|c|c|}
\hline$\chi^{2} / d f$ & $\mathrm{p}$ & RMR & GFI & NFI & IFI & CFI & RMSEA \\
\hline 2.183 & 0.000 & 0.038 & 0.925 & 0.947 & 0.949 & 0.947 & 0.048 \\
\hline
\end{tabular}

TABLE II. STRUCTURAL PATH ESTIMATES

\begin{tabular}{lcc}
\hline \multicolumn{1}{c}{ Relationship } & $\beta$ & $\mathrm{T}-\varpi \alpha \lambda \cup \varepsilon$ \\
\hline Emotional support-Physical QOL & 0.212 & 4.345 \\
Emotional support-Psychologioal QOL & 0.335 & 8.358 \\
Emotional support-Existential QOL & 0.274 & 5.620 \\
Informational support-Physical QOL & 0.569 & 10.256 \\
Informational support-Psychologioal QOL & 0.258 & 4.386 \\
Informational support-Existential QOL & 0.175 & 3.267 \\
Companionship-Physical QOL & 0.268 & 5.978 \\
Companionship-Psychological QOL & 0.357 & 7.286 \\
Companionship-Existential QOL & 0.329 & 7.107 \\
\hline
\end{tabular}

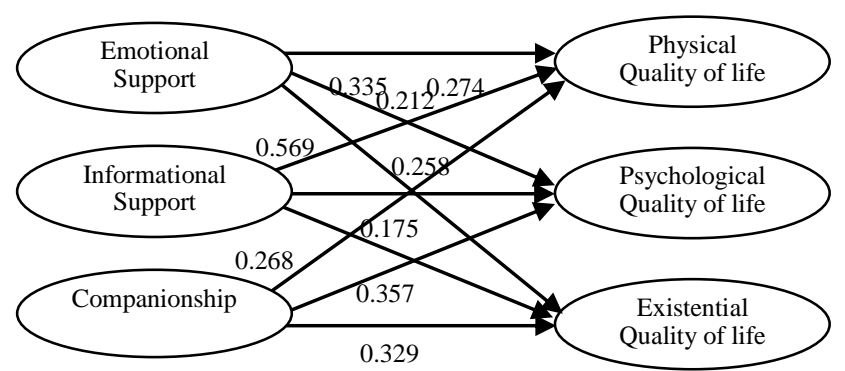

Figure 1. Path Model

\section{DISCUSSIONS}

On the basis of teasing apart online social support categories, we explored their links to patients' quality of life. For instance, we found evidence suggesting high quality of life tended to be associated with individuals who received 
different kinds of social support on the health care communities; however, the positive influence impacted by online social support on quality of life varies in degree and frequency across different subcategories. To summarize, the results of the empirical study indicated that the informational support, emotional support and companionship affect patients' quality of life in order of importance respectively. Web administrators should adjust and redesign if necessary the scopes and layouts of online health care communities in order to meet patients' needs to seek assistance, reconstruct interpersonal intercourse, and improve personal health state physically and psychologically. Such conclusions are critical to impelling reform and perfection of Chinese health care system, and producing deep and even new knowledge in health care service research and ultimately to the advancement of service science.

\section{ACKNOWLEDGMENT}

This research was financially supported by the National Natural Science Foundation of China (Grant No. 71202015, 71102032, 71232008, 71103013), Specialized Research Fund of Doctoral Program of University of Ministry of Education of China (Grant No. 20121102120022), Beihang University basic research program foundation, and China Postdoctoral Science Foundation (Grant No. 2012M510379). The corresponding author is Zheng Qiuying.

\section{REFERENCES}

[1] Castells, Manuel., The Rise of the Network Society, Volume I of The Information Age: Economy, Society and Culture. Massachusetts: Blackwell Publishing ,1996.

[2] Rheingold, Howard, The Virtual Community: Homesteading on the Electronic Fronter. MA: Addison Wesley Publishing Company ,1993.

[3] Ziebland, Sue, Alison Chapple, Carol Dumelow, Julie Evans, Suman Prinjha, and Linda Rozmovits, "How The Internet Affects Patients' Experience of Cancer: A Qualitative Study," British Medical Journal, 2004, 328 (7439), 564-569.

[4] Coulson, Neil S., "Receiving Social Support Online: An Analysis of A Computer-Mediated Support Group for Individuals Living with Irritable Bowel Syndrome," CyberPsychology and Behavior, 8 (6), 580-584,2005.

[5] Lamberg, Lynn, "Online Empathy for Mood Disorders-Patients Turn to Internet Support Groups," American Medical Association, 2003, 289 (23), 3073-3077.

[6] Bambina, Antonina, Online Social Support: the Interplay of Social Networks and Computer-mediated Communication. Youngstown, NY: Cambridge Press, 2007.

[7] Duchnick, Jennifer J., Elizabeth A. Letsch, and Glenn Curtiss, "Coping effectiveness training during acute rehabilitation of spinal cord injury/dysfunction: a randomized clinical trial," Rehabilitation Psychology, 2009, 54 (2), 123-132,2009.

[8] Rintala, Diana H., Mary E. Young, Karen A. Hart, Rebecca R. Clearman, and Marcus J Fuhrer, "Social Support and The Well-being of Persons with Spinal Cord Injury Living in The Community," Rehabilitation Psychology, 1992, 37 (3), 155-163.

[9] Elfström, Lundgren, M., Margareta Kreuter, Anna Rydén, Lars-Olof Persson, and Marianne Sullivan, "Effects of Coping On Psychological Outcome When Controlling for Background Variables: A Study of Traumatically Spinal Cord Lesioned Persons," Spinal Cord, 2002, 40 (8), 408-415
[10] Pollard, Clair and Paul Kennedy, "A Longitudinal Analysis of Emotional Impact, Coping Strategies and Post-Traumatic Psychological Growth Following Spinal Cord Injury: A 10-Year Review," British Journal of Health Psychology, 2007, 12 (3), 347-362.

[11] Agar, Elenor, Paul Kennedy, and Nigel S King, "The Role of Negative Cognitive Appraisals in Ptsd Symptoms Following Spinal Cord Injuries," Behavioural and Cognitive Psychotherapy, 2006, 34 (4), 437-452.

[12] McColl, Mary A., Robert Arnold, Susan Charlifue, and Kenneth Gerhart, "Social Support and Aging with A Spinal Cord Injury: Canadian and British Experiences," Topics in Spinal Cord Injury Rehabilitation, 2001, 6 (3), 83-101.

[13] Rintala, Diana H., Mary E. Young, Karen A. Hart, Rebecca R. Clearman, and Marcus J Fuhrer, "Social Support and The Well-being of Persons with Spinal Cord Injury Living in The Community," Rehabilitation Psychology, 1992, 37 (3), 155-163.

[14] Anderson, Catherine L. and Ritu Agarwal, "The Digitization of Healthcare: Boundary Risks, Emotion, and Consumer Willingness to Disclose Personal Health Information," Information Systems Research, 2011, 22 (3), 469-90.

[15] Barth, Jürgen, Sarah Schneider, and Roland von Känel, "Lack of Social Support in The Etiology and The Prognosis of Coronary Heart Disease: A Systematic Review and Meta-Analysis," Psychosomatic Medicine, 2010, 72 (3), 229-38.

[16] Salter, Katherine, Norine Foley, and Robert Teasell, "Social Support Interventions and Mood Status Post Stroke: A Review," International Journal of Nursing Studies, 2010, 47 (5), 616-25.

[17] Nausheen, Bina, Yori Gidron, Robert Peveler, and Rona Moss-Morris, "Social support and cancer progression: a systematic review," Journal of Psychosomatic Research, 2009, 67(5), 403-415.

[18] Wysocki, Tim and Peggy Greco, "Social Support and Diabetes Management in Childhood and Adolescence: Influence of Parents and Friends," Current Diabetes Reports, 2006 ,6(2), 117-122.

[19] Müller, Rolf, Claudio Peter, Alarcos Cieza, and Szilvia Geyh, "The Role of Social Support and Social Skills in People With Spinal Cord Injury-A Systematic Review of The Literature," Spinal Cord, 50 (2), 94-106.2012

[20] Cobb, Sidney, "Presidential Address-1976. Social Support as A Moderator of Life Stress," Psychosomatic Medicine, 38 (5), 300 $314,1976$.

[21] Sirgy, Joseph M., "Strategic Marketing Planning Guided by the Quality-of-Life (QOL) Concept," Journal of Business Ethics, 1996, 15 (3), 241-259.

[22] Clarke, Thomas, "Balancing The Triple Bottom Line: Financial, Social and Environmental Performance," Journal of General Management, 2001, 26 (4), 16-27.

[23] Kaplan, R.S. and Norton, D.P., "The Balanced Scorecard--measures that drive performance", Harvard Business Review, 1992, 70(1), 7179 .

[24] Tschopp, Daniel, "It's Time for Triple Bottom Line Reporting," The CPA Journal, 2003, 73 (12), 11-24.

[25] Sirgy, Joseph M. and Dong-Jin Lee, "Setting Socially Responsible Marketing Objectives: A Quality-of-Life Approach," European Journal of Marketing, 1996, 30 (5), 20-34.

[26] Sirgy, Joseph M., Coskun A. Samli and Lee H. Meadow, "The Interface between Quality of Life and Marketing: A Theoretical Framework," Journal of Marketing and Public Policy, 1982, 1 (1), 6984.

[27] Cohen, S. Robin, Balfour M. Mount, Michael G. Strobel, France Bui, "The McGill Quality of Life Questionnaire-a Measure of Quality of Life Appropriate for the People with Advanced disease. A Preliminary Study of Validity and Acceptability," Palliative Medicine, 1995, 9(3), 207-219.

[28] Li Jie and Hui Zhuang, "Advance in Epidemiology of Virus Hepatitis," Chinese Hepatology, 2011, 17 (1), 2-52. 
[29] Tichon, Jennifer G., and Margaret Shapiro., "The Process of Sharing Social Support in Cyberspace," CyberPsychology and Behavior, 2003, 6 (2), 161-170.

[30] Yin, Robert K., Case Study Research: Design and Methods. SAGE Publications, Incorporated, 1989.

[31] Cutrona, Carolyn E., and Julie A. Suhr, "Controllability of Stressful Events and Satisfaction With Spouse Support Behaviors," Communication Research, 1992, 19 (2), 154-174.

[32] Eichhorn, Kristen C., "Soliciting and Providing Social Support Over the Internet: An Investigation of Online Eating Disorder Support
Groups," Journal of Computer-Mediated Communication, 2008, 14 (1), 67-78.

[33] Schwarzer Ralf and Ute Schulz, "Social Support in Coping with Illness: The Berlin Social Support Scales (BSSS)," Diagnostica, 2003, 49 (2), 73-82.

[34] Hau, Kit-Tai and Herbert W. Marsh, "The Use of Item Parcels in Structural Equation Modeling: Non-Normal Data and Small Sample Sizes," British Journal of Mathematical Statistical Psychology, 2004, 57 (2), 327-351. 